\title{
Labeler Regulatory Contact for FDA
}

National Cancer Institute

\section{Source}

National Cancer Institute. Labeler Regulatory Contact for FDA. NCI Thesaurus. Code C101701.

The representative of a labeler company who is the authorized channel for communication between the company and the FDA. 